\title{
Evoluzione storica della farmacoutilizzazione degli anti-ipertensivi in Italia (1988-1998)
}

Enrico Bologna*, Pierluigi Russo ${ }^{\circ}$,Sabrina Licata ${ }^{\circ}$ Liliana Civalleri $^{*}$, Luciano Caprino $^{\circ}$

\begin{abstract}
The aim of this study was to perform an historical evaluation of pharmacoutilization of main antihypertensive drug classes in Italy between 1988 and 1998.

There were analysed numbers of packs and costs for diuretics, beta-blockers, central and peripheral antihypertensive drugs (CPAD), calcium-channel blockers (CCB), angiotensin-converting enzyme (ACE) inhibitors and angiotensin-II (AII) receptor antagonists. The amount of defined-daily-doses (DDDs) per preparation was calculated by dividing the annual number of packs by respective DDD value. The number of DDDs was grouped according to the second level of the anatomic-therapeutic-chemical (ATC) classification. The amount of annual expenditures for each ATC level was converted to current monetary values. To calculate the annual cost per DDD, the expenditure was divided by respective number of DDDs.

From 1988 to 1998, total number of DDDs of antihypertensive drugs grew from 193.657 .092 to 396.140 .967 with an increase of $105 \%$, while the expenditure grew from 40.886 .385 to 126.102 .362 , with an increase of $208 \%$. Comparing data of 1990 and 1998, the DDDs of antihypertensive drugs increased of about 79\%, the expenditure for these drugs increased of about $115 \%$, while the global pharmaceutical expenditure increased only of $34 \%$.

Taking each drug class separately, CCB and ACE-inhibitors are the classes with the higher number of DDDs and the higher expenditure; while alpha-receptor blockers, ACE-inhibitors and AII- antagonists are the classes with the higher cost per DDD.

In conclusion this study describes a substantial modification of pharmacoutilization of antihypertensive drugs in Italy along a ten-years span. The agreement between utilization trends and indications of international treatment guidelines, as well as the economic impact on drugs expenditure have been discussed.
\end{abstract}

Farmeconomia e percorsi terapeutici 2003; 4 (Suppl 1): 35-44

\section{INTRODUZIONE}

In Italia, come in altri paesi occidentali, le malattie cardiovascolari rappresentano la causa più frequente di morte (oltre il $36 \%$ ), precedendo a grande distanza quelle dell'apparato respiratorio (poco più del $5 \%$ ) e dell' apparato digerente (circa il 4\%) e rimanendo lontane dalle malattie neoplastiche (circa il 25\%) [1].

Gran parte delle malattie cardiovascolari riconoscono nell'ipertensione arteriosa un fattore causale o concausale; in effetti la correlazione esistente tra pressione arteriosa e rischio cardiovascolare è ben documentata, stretta, continua, indipendente, predittiva ed eziologicamente significativa [2]. Dal punto di vista epidemiologico, si stima che la prevalenza dell'ipertensione arteriosa sia variabile tra il
$27,6 \%$ nell' America del Nord e il 44,2\% in Europa $(37,7 \%$ in Italia) [3]. In altri termini, considerato che circa un terzo dei soggetti adulti e circa il $60 \%$ degli anziani che vivono nei paesi occidentali sono ipertesi, si può correttamente affermare che l'ipertensione arteriosa è la più diffusa condizione morbosa per la quale sia disponibile un efficace trattamento farmacologico [4]. La somministrazione di farmaci anti-ipertensivi si è dimostrata infatti capace di ridurre in misura significativa il rischio di ictus cerebrale, di eventi coronarici, di insufficienza cardiaca e di progressione dell'insufficienza renale. All'evoluzione nella capacità di riconoscere e trattare l'ipertensione viene del resto attribuito il maggior merito della forte riduzione della letalità per ictus cerebrale (circa il 60\%) e per malattia coronarica (circa il 50\%) osservata negli ultimi trenta anni.
*Società Italiana per studi di Economia ed Etica sul farmaco e sugli interventi terapeutici (SIFEIT)

'Dipartimento di Fisiologia Umana e Farmacologia, Università

"La Sapienza”, Roma 
Poiché la distribuzione della pressione arteriosa nella popolazione è di tipo continuo, il valore soglia per la definizione di ipertensione è stato arbitrariamente stabilito in $=140 \mathrm{mmHg}$ per la sistolica e $=90 \mathrm{mmHg}$ per la diastolica. Ma proprio per la linearità della correlazione tra valori pressori e rischio cardiovascolare, dal 1997 gli estensori del sesto Rapporto del Joint National Committee on Prevention, Detection, Evaluation and Treatment of the High Blood Pressure (JNC) dei National Institutes of Health statunitensi [4] hanno proposto una più articolata classificazione dell'ipertensione arteriosa per i soggetti adulti, spostando verso il basso i valori desiderabili. Tale impostazione è stata mantenuta nel rapporto pubblicato nel 2003 (Tabella 1), dove è confermata come pressione arteriosa ottimale quella inferiore a $120 / 80 \mathrm{mmHg}$ [5] .

Questa riclassificazione della pressione arteriosa, che mette in evidenza l'opportunità di ottenere valori pressori inferiori rispetto a quelli fino a pochi anni fa considerati "normali", allarga la platea dei soggetti che possono richiedere l'uso di farmaci anti-ipertensivi. Altro elemento che tende a determinare una analoga conseguenza è rappresentato dalla crescente diffusione del trattamento dell'ipertensione anche negli anziani. In questi ultimi l'aumento della pressione arteriosa è stato a lungo considerato una manifestazione del processo di invecchiamento anziché una malattia e, come tale, fino ad epoca recente vi era una diffusa riluttanza a trattarlo o, quanto meno, a trattarlo con modalità e con obiettivi analoghi a quelli adottati per gli ipertesi più giovani. Questo atteggiamento era suggerito dal timore che la riduzione della pressione arteriosa potesse favorire episodi di ipotensione ortostatica e determinare fenomeni ischemici cerebrali o coronarici.

Non vi è dubbio sul fatto che la pressione arteriosa si modifichi con l'età: il Framingham
Study, nel quale sono state studiate le variazioni pressorie legate all'età in soggetti normotesi e in ipertesi non trattati seguiti per periodi fino a 30 anni, ha permesso di rilevare che la pressione sistolica aumenta in modo lineare dai 30 agli 84 anni, con un concomitante aumento della pressione diastolica e della pressione media fino ai 50-60 anni. Da questa età la pressione diastolica tende a ridursi e la pressione differenziale ad aumentare, mentre la pressione media si stabilizza [6]. Ma se è vero che l'ipertensione arteriosa accompagna così spesso l'invecchiamento da apparire come una caratteristica dell' età avanzata, è ugualmente vero che, anche in questa fase della vita, essa predispone a tutte le principali complicazioni cardiovascolari, e che anche negli anziani tali complicazioni possono essere efficacemente prevenute con il trattamento anti-ipertensivo. Anche il trattamento dell'ipertensione sistolica isolata dell'anziano è in grado di ridurre la morbilità e la letalità; ciò è stato confermato da numerose osservazioni, in base alle quali si può ritenere accertato che l'ipertensione sistolica isolata, così frequente negli anziani, ha un valore predittivo nei confronti di eventi vascolari uguale se non superiore rispetto a quello della ipertensione diastolica. Tutti queste recenti acquisizioni sono state fatte proprie nelle linee guida dell'Organizzazione Mondiale della Sanità del 1999 [7].

Anche per quanto riguarda la fascia di età degli ultraottantenni, quella che mostra attualmente la maggiore espansione, le metanalisi relative alla quota degli ipertesi anziani arruolati in numerosi studi clinici controllati (SHEP [8], Syst-Eur[9], Syst-China[10], EWPHE[11], STOP Hypertension[12], MRC[13], STONE[14]) hanno dimostrato che la riduzione farmacologica della pressione arteriosa determina una significativa riduzione del rischio di complicazioni, benché sembri non influenzare la letalità cardiovascolare (Tabella 2) $[15,16]$.

\section{Tabella 1}

Classificazione della pressione arteriosa in adulti secondo il Joint National Committee on Prevention, Detection, Evaluation, and Treatment of High Blood Pressure (JNC) $[4,5]$.

\begin{tabular}{lcccc}
\hline Pressione (mmHg) & \multicolumn{2}{c}{ JNC 1997 } & \multicolumn{2}{c}{ JNC 2003 } \\
& Sistolica & Diastolica & Sistolica & Diastolica \\
\hline Ottimale & $<120$ & $<80$ & - & - \\
Normale & $<130$ & $<85$ & $<120$ & $<80$ \\
Alta normale* & $130-139$ o & $85-89$ & $120-139$ o & $80-89$ \\
$\begin{array}{l}\text { Ipertensione } \\
\text { Stadio 1 } \\
\text { Stadio 2 } \\
\text { Stadio 3 }\end{array}$ & $140-159$ o $90-99$ & & $140-159$ o $90-99$ \\
\hline
\end{tabular}

* definita come pre-ipertensione nel JNC 2003 [5]

** nella classificazione JNC 2003 sono considerati solamente due stadi di ipertensione [5] 
Come sopra accennato, $\mathrm{i}$ benefici del trattamento anti-ipertensivo sono stati confermati anche in soggetti con ipertensione sistolica isolata; in questi ipertesi, che sono in gran parte anziani, la riduzione della pressione sistolica si accompagna ad una significativa riduzione non solo del rischio di complicanze, ma anche della letalità per cause cardiovascolari. In particolare, una metanalisi condotta su 15.693 soggetti con ipertensione sistolica isolata ha evidenziato che il numero di soggetti da trattare per prevenire un evento cardiovascolare maggiore entro cinque anni è più basso negli ipertesi ultrasettantenni rispetto a quelli di età inferiore [17].

Le conclusioni di queste e di altre numerose osservazioni sono state fatte proprie dai già citati rapporti del JNC [4,5], nei quali si raccomanda di trattare gli anziani secondo modalità (dosi, farmaci e associazioni) analoghe a quelle seguite per gli ipertesi più giovani, con l'unica differenza rappresentata dal consiglio di iniziare il trattamento con dosi ridotte.

All'abbassamento dei valori pressori desiderabili e alle dimostrazioni dell' utilità del trattamento anti-ipertensivo anche nella popolazione anziana si aggiunge la certezza che questo trattamento farmacologico è uno degli interventi più favorevoli, in termini di rapporto costo-efficacia, tra quelli disponibili per ridur- re il rischio cardiovascolare. Queste acquisizioni porteranno certamente ad un rilevante allargamento del numero dei soggetti candidati alla terapia con farmaci anti-ipertensivi. Il crescente numero di individui potenzialmente eleggibili per il trattamento anti-ipertensivo apre nuovi scenari di valutazione farmacoeconomica, in grado di considerare sia i problemi connessi con un'efficiente allocazione degli antiipertensivi sulla base della continuità al trattamento [18], sia le esigenze di contenimento della spesa farmaceutica, al fine di definire le migliori strategie da attuare in quest'ambito. Ciò soprattutto in considerazione del crescente costo delle nuove molecole introdotte, il cui uso tende a sostituire quello degli antiipertensivi più datati (Figura 1).

La dimensione del problema emerge chiaramente dai dati relativi al 2002 resi pubblici dall'Osservatorio Nazionale sull'Impiego dei Medicinali del Ministero della Salute [19]. Nello scorso anno la spesa farmaceutica lorda ha raggiunto un totale di 12.644 milioni di euro (218 euro pro capite), di cui 11.722 milioni di euro a carico del SSN. Il 24,7\% di quest'ultima cifra, pari a 2.892 milioni di euro, è stata la spesa per farmaci utilizzati nel trattamento dell'ipertensione arteriosa. Tuttavia, sottraendo da tale cifra la parte di spesa relativa all'impiego dei medesimi farmaci in indicazioni differenti dal-

\begin{tabular}{|c|c|c|c|c|c|c|c|c|}
\hline STUDIO & $\begin{array}{l}\text { N età } \\
\text { media }\end{array}$ & $\begin{array}{l}\text { PAS } \\
\text { PAD }\end{array}$ & $\begin{array}{l}\text { Terapia fase } 1 \\
\text { Terapia fase } 2\end{array}$ & $\begin{array}{r}\Delta \% \\
\text { Ictus }\end{array}$ & $\begin{array}{c}\Delta \% \\
\mathrm{EC}\end{array}$ & $\begin{array}{c}\Delta \% \\
\mathrm{E} C \mathrm{~V}\end{array}$ & $\begin{array}{c}\Delta \% \\
\mathrm{LCV}\end{array}$ & $\begin{array}{c}\Delta \% \\
\text { LT }\end{array}$ \\
\hline $\begin{array}{l}\text { SHEP } \\
\text { IA sistolica isolata }\end{array}$ & $\begin{array}{l}4736 \\
71.6\end{array}$ & $170 / 77$ & $\begin{array}{l}\text { Clortalidone } \\
\text { Atenololo }\end{array}$ & $-36^{*}$ & -27 & $-32 *$ & -20 & -13 \\
\hline $\begin{array}{l}\text { Syst-Eur } \\
\text { IA sistolica isolata }\end{array}$ & $\begin{array}{l}4695 \\
70.2\end{array}$ & $174 / 85$ & $\begin{array}{l}\text { Nitrendipina } \\
\text { Enalapril }\end{array}$ & $-42 *$ & $-26^{*}$ & $-31^{*}$ & -27 & -14 \\
\hline $\begin{array}{l}\text { Syst-China } \\
\text { IA sistolica isolata }\end{array}$ & $\begin{array}{c}2394 \\
66.5\end{array}$ & $171 / 86$ & $\begin{array}{l}\text { Nitrendipina } \\
\text { Captopril }\end{array}$ & $-38 *$ & -37 & $-37^{*}$ & $-39 *$ & $-39 *$ \\
\hline $\begin{array}{l}\text { EWPHE } \\
\text { IA sisto-diastolica }\end{array}$ & $\begin{array}{r}840 \\
72.0\end{array}$ & $182 / 101$ & $\begin{array}{l}\text { HTC+Triamterene } \\
\alpha \text {-metildopa }\end{array}$ & -32 & $-47 *$ & $-38 *$ & $-27^{*}$ & -9 \\
\hline $\begin{array}{l}\text { STOP-hypertension } \\
\text { IA sistolica o diastolica }\end{array}$ & $\begin{array}{l}1627 \\
76.0\end{array}$ & $195 / 102$ & $\begin{array}{l}\text { Diuretico o } \beta \text {-bloccante } \\
\text { Diuretico o } \beta \text {-bloccante }\end{array}$ & $-47 *$ & -13 & $-40^{*}$ & - & -43 \\
\hline $\begin{array}{l}\text { Coop\&Warrender } \\
\text { IA sistolica o diastolica }\end{array}$ & $\begin{array}{l}884 \\
68.7\end{array}$ & $196 / 99$ & $\begin{array}{l}\text { Atenololo } \\
\text { Diuretico }\end{array}$ & $-42 *$ & +3 & - & -22 & -3 \\
\hline $\begin{array}{l}\text { MRC } \\
\text { IA sistolica o diastolica }\end{array}$ & $\begin{array}{l}4396 \\
70.0\end{array}$ & $185 / 91$ & $\begin{array}{l}\text { Diuretico o } \beta \text {-bloccante } \\
\text { Diuretico o } \beta \text {-bloccante }\end{array}$ & $-25^{*}$ & -19 & $-17^{*}$ & -9 & -3 \\
\hline $\begin{array}{l}\text { Stone } \\
\text { IA sistolica o diastolica }\end{array}$ & $\begin{array}{l}1632 \\
66.0\end{array}$ & $168 / 100$ & $\begin{array}{l}\text { Nifedipina } \\
\text { Captopril }\end{array}$ & $-57 *$ & -6 & $-60^{*}$ & -26 & $-45^{*}$ \\
\hline
\end{tabular}

$E C=$ eventi coronarici; $E C V=$ eventi cardiovascolari; $L C V=$ letalità cardiovascolare; $L T=$ letalità totale

* Variazioni statisticamente significative

\section{Tabella 2}

Studi clinici controllati condotti su anziani con ipertensione arteriosa 


\section{Figura 1}

Costo annuale della terapia anti-ipertensiva con le diverse molecole disponibili (costi dei farmaci aggiornati al febbraio 2003)

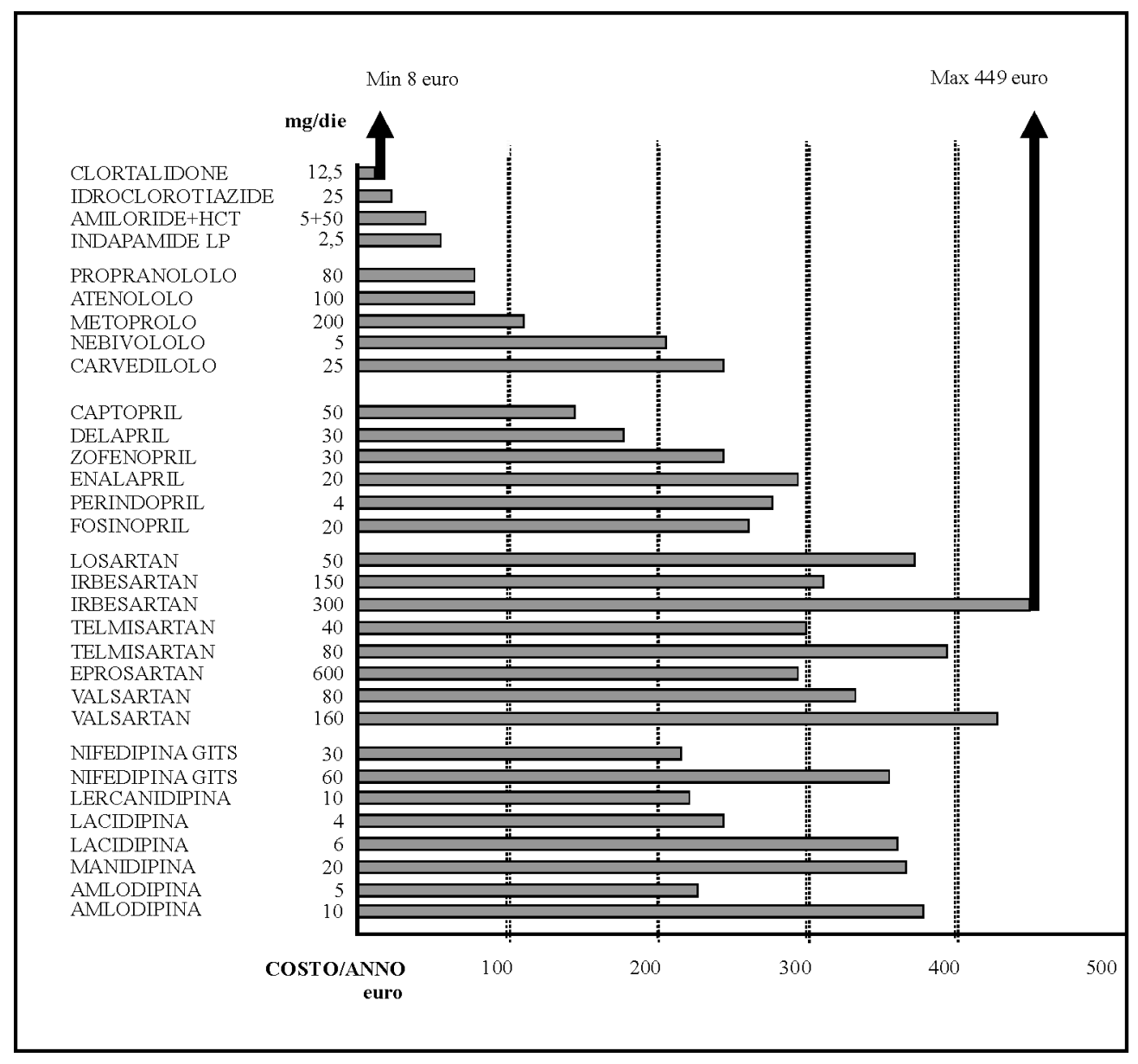

l'ipertensione arteriosa, quota in genere valutabile intorno al $15 \%$, si può concludere che per il trattamento farmacologico dell'ipertensione arteriosa nel 2002 sono stati spesi circa 2.458 milioni di euro, pari al 19,4\% della spesa farmaceutica totale.

Un dato di spesa così elevato (circa un quinto della spesa farmaceutica pubblica totale) per un'unica condizione morbosa merita di essere attentamente valutato, soprattutto in conside- razione delle enormi differenze di prezzo tra le diverse molecole anti-ipertensive: un anno di trattamento anti-ipertensivo può costare da meno di 10 euro, se si utilizza un diuretico, a circa 450 euro, se si impiega un inibitore recettoriale dell' angiotensina II (Figura 1).

Scopo del presente studio è quello di offrire una descrizione dell'evoluzione storica in un recente passato (1988-1998) della farmacoutilizzazione degli anti-ipertensivi in Italia.

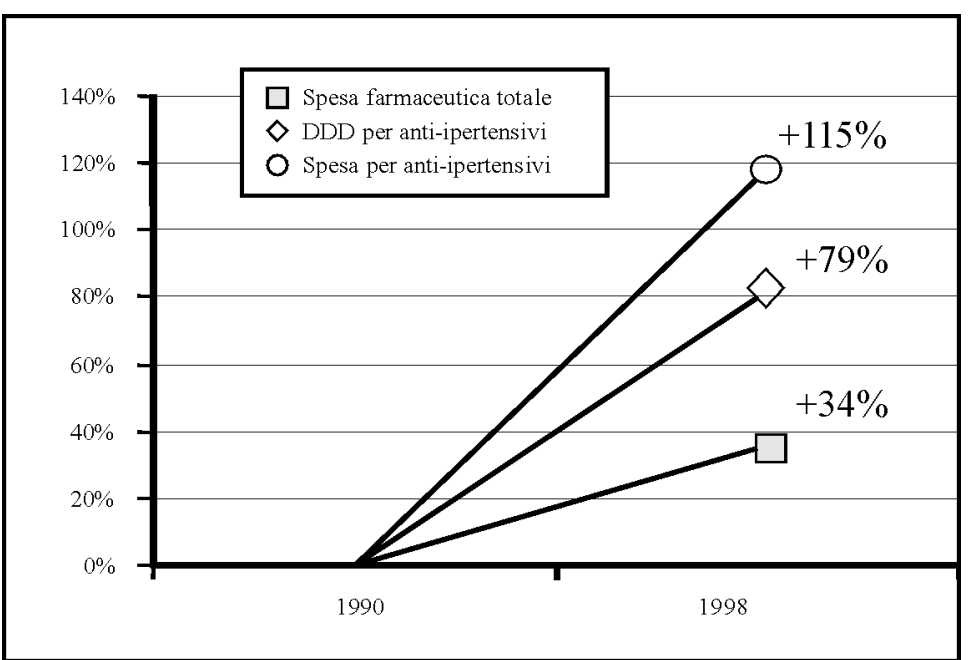

\section{Figura 2}

Variazioni percentuali del numero di DDD, della spesa farmaceutica globale e per anti-ipertensivi, nel 1998 rispetto al 1990

\section{MATERIALIEMETODI}

A tale scopo sono stati analizzati i dati relativi al numero di confezioni vendute e alla spesa per farmaci anti-ipertensivi nel periodo 19881998 [20], come pure alla spesa farmaceutica pubblica nel periodo 1990-2002[21]. Benché il numero di confezioni vendute non sia un indicatore dell'effettiva utilizzazione di un farmaco da parte del paziente, ne rappresenta un valido surrogato, già ampiamente adottato in letteratura.

I farmaci anti-ipertensivi presi in considerazione sono tutti quelli compresi nelle seguenti categorie ATC:

- C02 Anti-ipertensivi ad azione centrale e periferica (clonidina, metildopa, reserpina, bloccanti del recettore alfa-adrenergico, 
indoramina, urapidil, diidroergotossina e ketanserina);

- C03 Diuretici e risparmiatori di potassio;

- C07 Beta-bloccanti;

- C08 Calcio-antagonisti;

- C09 Sostanze ad azione sul sistema reninaangiotensina: antagonisti dell'enzima di conversione dell' angiotensina (ACEinibitori) e antagonisti del recettore per l'angiotensina II (antagonisti AII).

L'analisi dei consumi è stata condotta in accordo con la procedura precedentemente descritta basata sulla dose giornaliera definita (DDD) [22]. In particolare, è stata calcolata la quantità in $\mathrm{mg}$ di principio attivo presente in ogni confezione e successivamente tale valore è stato moltiplicato per il numero di confezioni vendute in ogni anno. È stato quindi calcolato il numero di DDD per anno dividendo $i$ mg totali consumati ogni anno per il corrispondente valore di DDD della specifica forma farmaceutica. Infine è stato calcolato il numero complessivo di DDD consumate all'anno per ognuna delle categorie ATC prese in considerazione.

Per quanto riguarda i limiti della metodologia di analisi dei consumi di farmaci basata sulla DDD, si rimanda il lettore a precedenti pubblicazioni che hanno già affrontato la problematica in modo dettagliato [22].

In modo analogo sono stati calcolati i valori di spesa annuale per ogni categoria ATC considerata, partendo dalla spesa annuale per ogni confezione. Tali valori sono stati tradotti in euro (attraverso il tasso di conversione di 1 euro $=1.936,27$ lire) e attualizzati al settembre 2002.

\section{RISULTATI}

Tra il 1988 e il 1998 il numero di DDD di farmaci anti-ipertensivi è passato da 193.657.092 a 396.140 .967 con un incremento del $105 \%$, mentre nello stesso periodo la spesa per questi farmaci è passata da 40.886.385 euro (spesa attualizzata 69.540.000 euro) a 126.102.362 euro (spesa attualizzata 138.490 .000 euro) con un incremento del $208 \%$, valore che si riduce al $99 \%$ se si considerano i valori di spesa attualizzati.

Nella Figura 2 è mostrata una valutazione comparativa delle variazioni del numero di DDD e della spesa per farmaci anti-ipertensivi, rispetto alla spesa farmaceutica complessiva, nell'intervallo temporale compreso tra il $1990 \mathrm{e}$ il 1998. Mentre il numero delle DDD per antiipertensivi è aumentato di circa il 79\%, la spesa per questi farmaci è aumentata del $115 \%$, a fronte di un incremento della spesa farmaceutica globale del $34 \%$. In altri termini, nel periodo considerato, la spesa per la terapia farmacologica dell'ipertensione arteriosa è cresciuta con un ritmo superiore al triplo rispetto alla spesa farmaceutica globale.

Nella Figura 3 si vedono gli andamenti delle DDD nell'intervallo temporale che va dal 1988 al 1998, separatamente per preparazioni farmaceutiche monocomponente (Figura 3a) e in associazione (Figura 3b).

Nella Figura 3a è possibile rilevare come i Ca-antagonisti e gli ACE-inibitori presentino un andamento crescente, con una pendenza simile a quella degli antagonisti AII negli ultimi due anni di osservazione. Per quanto riguarda
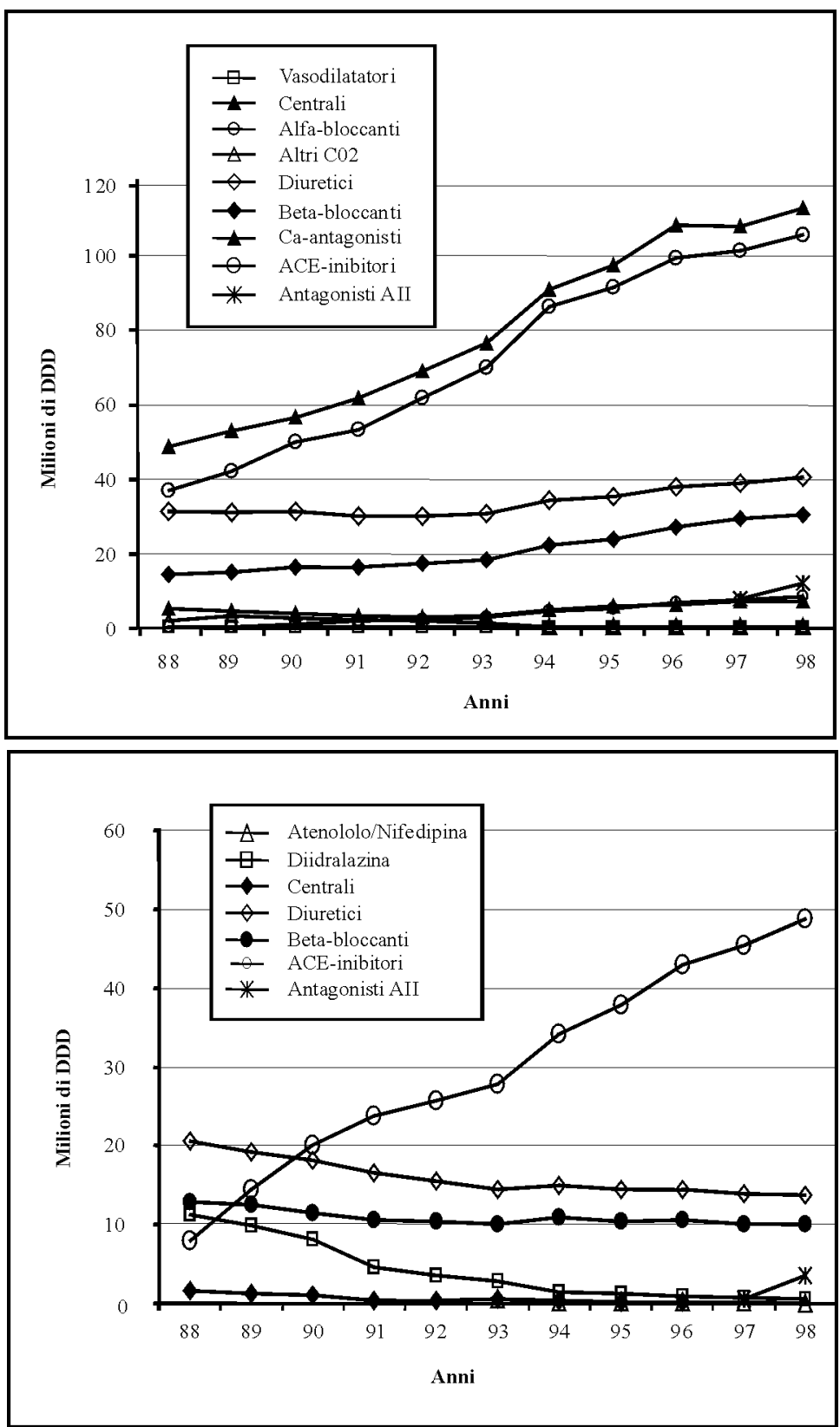

Figura 3a - 3b

Andamenti del numero di DDD per farmaci anti-ipertensivi tra il 1988 e il 1998, separatamente per preparazioni farmaceutiche monocomponente (3a) e in associazione (3b) 
i diuretici, il numero di DDD rimane stazionario fino al 1993, successivamente presenta un lento ma costante aumento; un andamento analogo si è evidenziato anche per i beta-bloccanti. In sintesi, Ca-antagonisti e ACE-inibitori sono emersi come gli anti-ipertensivi più importanti, in termini di DDD vendute, nel periodo di tempo considerato.

La Figura 3b mette in evidenza gli andamenti del numero di DDD delle associazioni di
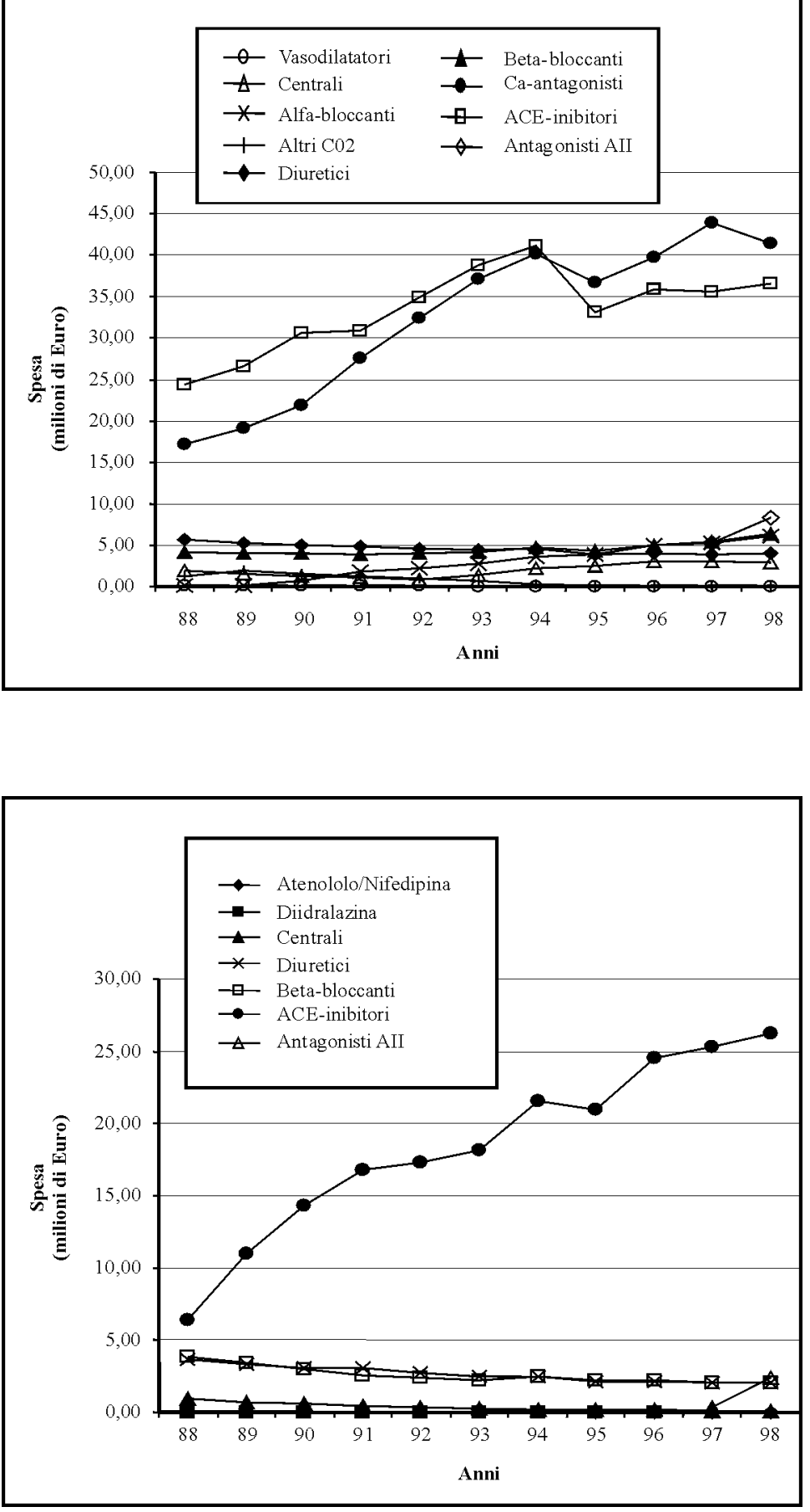

Figura 4a - 4b

Andamenti della spesa per farmaci anti-ipertensivi tra il 1988 e il 1998 (attualizzata al 2002), separatamente per preparazioni farmaceutiche monocomponente (4a) $e$ in associazione (4b) anti-ipertensivi, dominati dalla crescita degli ACE-inibitori in associazione con un diuretico che, in corrispondenza del 1993, presentano un modesto rallentamento attribuibile alla riduzione dei consumi delle associazioni a base di captopril.

Considerando complessivamente la classe degli ACE-inibitori, il totale delle DDD vendute passa da 44.745 .610 nel 1988 a 154.554 .164 nel 1998, con un aumento pari al $245 \%$, posizionandoli al primo posto tra i farmaci antiipertensivi, come DDD vendute.

Per quanto riguarda gli anti-ipertensivi ad azione centrale in associazione con diuretico, questi mostrano una riduzione del numero di DDD vendute che inizia intorno ai primi anni ' 90 , e che è soprattutto imputabile alla caduta dell'uso di formulazioni farmaceutiche a base di reserpina. Le associazioni costituite da diuretici e risparmiatori di potassio sono apparse in lento calo in tutto il periodo considerato.

Infine, gli antagonisti recettoriali AII, nonostante siano entrati in uso solo negli ultimi due anni del periodo considerato, mostrano un andamento in netta crescita con un aumento delle DDD di circa il $94 \%$.

Analogamente al numero di DDD vendute, l'andamento della spesa per preparazioni farmaceutiche monocomponente (Figura 4a) e in associazione (Figura 4b) è caratterizzato dal primato dei Ca-antagonisti e degli ACE-inibitori, tuttavia il trend dei primi rispetto ai secondi è diverso rispetto a quello registrato per le DDD vendute. In particolare (Figura 4a), i Ca-antagonisti che, nel 1988, partono da un valore di spesa nettamente inferiore a quello degli ACEinibitori (17.140.000 euro vs 24.390.000 euro), raggiungono questi ultimi nel 1994, per poi superarli negli anni successivi. La modificazione della pendenza di crescita nel tempo dei Caantagonisti è dovuta alla comparsa di DDD per amlodipina a partire dal 1990. Per quanto concerne gli ACE-inibitori, la spesa raggiunge un picco massimo nel 1994 (41.090.000 euro). Sia i Ca-antagonisti sia gli ACE-inibitori nel 1995 mostrano una brusca riduzione della spesa non associata ad una contemporanea riduzione del numero di DDD vendute, seguita poi da una lenta crescita negli ultimi anni del periodo considerato. La consistente modificazione della spesa negli anni 1994-95 appare essere in relazione con l'introduzione del nuovo prontuario farmaceutico nazionale nel gennaio del 1994, caratterizzato dalla regolamentazione dell'uso dei farmaci sia su criteri di restrizione in rapporto all'efficacia terapeutica (fasce) sia in rapporto ad aspetti economici (costo massimo per ciclo di terapia) [23].

Con variazioni di minor entità rispetto ai Ca-antagonisti e agli ACE-inibitori, la spesa per alfa-bloccanti ha mostrato un progressivo 
aumento a partire dal 1990, anno in cui è stata introdotta in Italia la dossazosina. La spesa per farmaci ad azione centrale, inizialmente in calo, presenta un progressivo aumento a partire dal 1993, quando è stata introdotta in commercio la clonidina transdermica (TTS). Per quanto concerne la spesa per diuretici, betabloccanti e vasodilatatori, si registrano bassi volumi di spesa e modeste variazioni.

Infine, netto è l'aumento della spesa riguardante gli antagonisti AII che va da 5.280.000 euro nel 1997 a 8.290.000 euro nel 1998 con un incremento del $90 \%$ in un anno.

Per quanto riguarda la spesa per le associazioni (Figura 4b), la curva relativa a quelle contenenti un ACE-inibitore si distacca completamente dalle altre. La spesa, che nel 1988 è pari a circa 6.370.000 euro, sale a 26.240.000 euro nel 1998, con un andamento che vede una transitoria riduzione solo nel 1995 (effetto dell' introduzione del nuovo prontuario farmaceutico nazionale). Presa complessivamente, la classe dei farmaci contenenti un ACEinibitore passa da una spesa di circa 30.760.000 euro nel 1988 a 66.750.000 euro nel 1998 con un incremento di circa il $117 \%$.

Ancora maggiore è l'aumento della spesa per gli antagonisti AII in associazione, che dal 1997 al 1998 passa da 310.000 euro a 2.360 .000 euro con un incremento di oltre 7 volte.

Nelle Figure 5a e 5b sono riportati i costi attualizzati per DDD per ogni singola classe. Relativamente alle preparazioni farmaceutiche monocomponente (Figura 5a), gli alfa-bloccanti risultano quelli con il maggior costo per DDD, mostrando un fortissimo incremento tra il 1989 ( 0,25 euro) e il 1990 ( 0,85 euro), con un valore massimo nel 1991 pari a 0,96 euro; risultando in assoluto il costo per DDD più alto nel periodo considerato. Un aumento così marcato potrebbe essere messo in relazione con l'introduzione in commercio di due nuove molecole, terazosina (1990) e dossazosina (1991). Successivamente il costo per DDD scende progressivamente sino a raggiungere il valore di 0,70 euro, valore che risulta comunque il più alto rispetto a quello delle altre classi di antiipertensivi e di poco superiore a quello degli antagonisti AII pari a 0,68 euro. Il costo per DDD degli anti-ipertensivi ad azione centrale presenta un progressivo aumento, che si accentua dal 1993 in poi, in coincidenza con l'immissione in commercio della clonidina TTS. Al contrario, i vasodilatatori mostrano una lenta e progressiva diminuzione sino al 1994, anno in cui la spesa della cadralazina passa da 34.035 euro a 13.996 euro; tale decremento incide sull'andamento del costo per DDD di questa classe. Successivamente, però, si osserva un aumento che ha coinciso con il ritiro dal commercio della meno costosa diidralazina.
Diuretici e beta-bloccanti sono le classi di farmaci con il costo per DDD più stabile nel tempo; inoltre i diuretici rappresentano i farmaci con il minor costo per DDD in tutto il periodo considerato, variando tra 0,10 euro e 0,18 euro. I Ca-antagonisti mostrano un aumento del costo per DDD sino al 1993; dal 1994 il costo tende a scendere sino quasi a raggiungere il valore iniziale $(0,35$ euro vs 0,37 euro valore finale).

Gli ACE-inibitori presentano il valore massimo del costo per DDD nel 1988 (0,66 euro) e successivamente evidenziano una progressiva diminuzione, che diventa più marcata tra il 1994 e il 1995, raggiungendo un valore di 0,27 euro nel 1998.

Per quanto riguarda il costo per DDD delle associazioni di farmaci anti-ipertensivi (Figura $5 b)$, quelle con ACE-inibitori hanno il valore massimo nel 1988 (0,81 euro), che diminuisce successivamente fino a raggiungere un valore di 0,54 euro nel 1998.

Gli anti-ipertensivi ad azione centrale in associazione mostrano un progressivo aumento del costo per DDD, caratterizzato da un incremento più evidente tra il 1993 e il 1994, in coincidenza con una diminuzione delle DDD vendute delle associazioni con reserpina.

Le preparazioni in associazione con diuretici e rispettivamente con beta-bloccanti sono le classi di farmaci con il costo per DDD più stabile nel tempo. Inoltre, l'associazione con diidralazina risulta essere quella con il più basso costo per DDD, variando tra 0,02 euro e 0,01 euro nel 1996, superato il quale non compaiono più DDD vendute a causa del suo ritiro dal commercio.

L'associazione atenololo-nifedipina, che entra in commercio dal 1993, presenta un costo per DDD tra i più alti ( 0,30 euro), dopo quello degli ACE-inibitori e degli antagonisti AII, rimanendo costante negli anni successivi.

Infine gli antagonisti AII in associazione presentano il costo per DDD più elevato, con valori intorno a 0,68 euro, risultando nel 1998 l'anti-ipertensivo con il costo per DDD più elevato, insieme agli alfa-bloccanti e agli antagonisti AII in preparazione farmaceutica monocomponente.

\section{DISCUSSIONE}

In Italia, come in tutti i paesi occidentali, l'ipertensione arteriosa è molto diffusa e il numero di candidati al trattamento con farmaci anti-ipertensivi, da anni in costante aumento, è destinato ad aumentare ulteriormente. Questa previsione trova una conferma nei dati del National Health and Nutrition Examination Survey (NAHNES), riportati nel JNC 2003 [5], 
che ha valutato l'andamento tra il 1976 e il 2000 della percentuale di soggetti ipertesi consapevoli di essere tali (passata dal $51 \%$ al $70 \%$ ), di quelli in trattamento farmacologico (aumentata dal $31 \%$ al $59 \%$ ) e di coloro nei quali tale trattamento ha permesso di normalizzare i valori pressori (aumentata dal 10\% al 34\%). Come si vede, anche nella rilevazione più recente, circa un terzo degli ipertesi ignora la propria malattia, meno del $60 \%$ segue un trattamento e solo in un terzo dei pazienti la pressione arteriosa è tenuta a valori accettabili grazie alla terapia.

Il prevedibile progressivo miglioramento del controllo dell'ipertensione arteriosa nella popolazione comporterà necessariamente un rilevante aumento del consumo di farmaci antiipertensivi. Questo processo del resto è già in
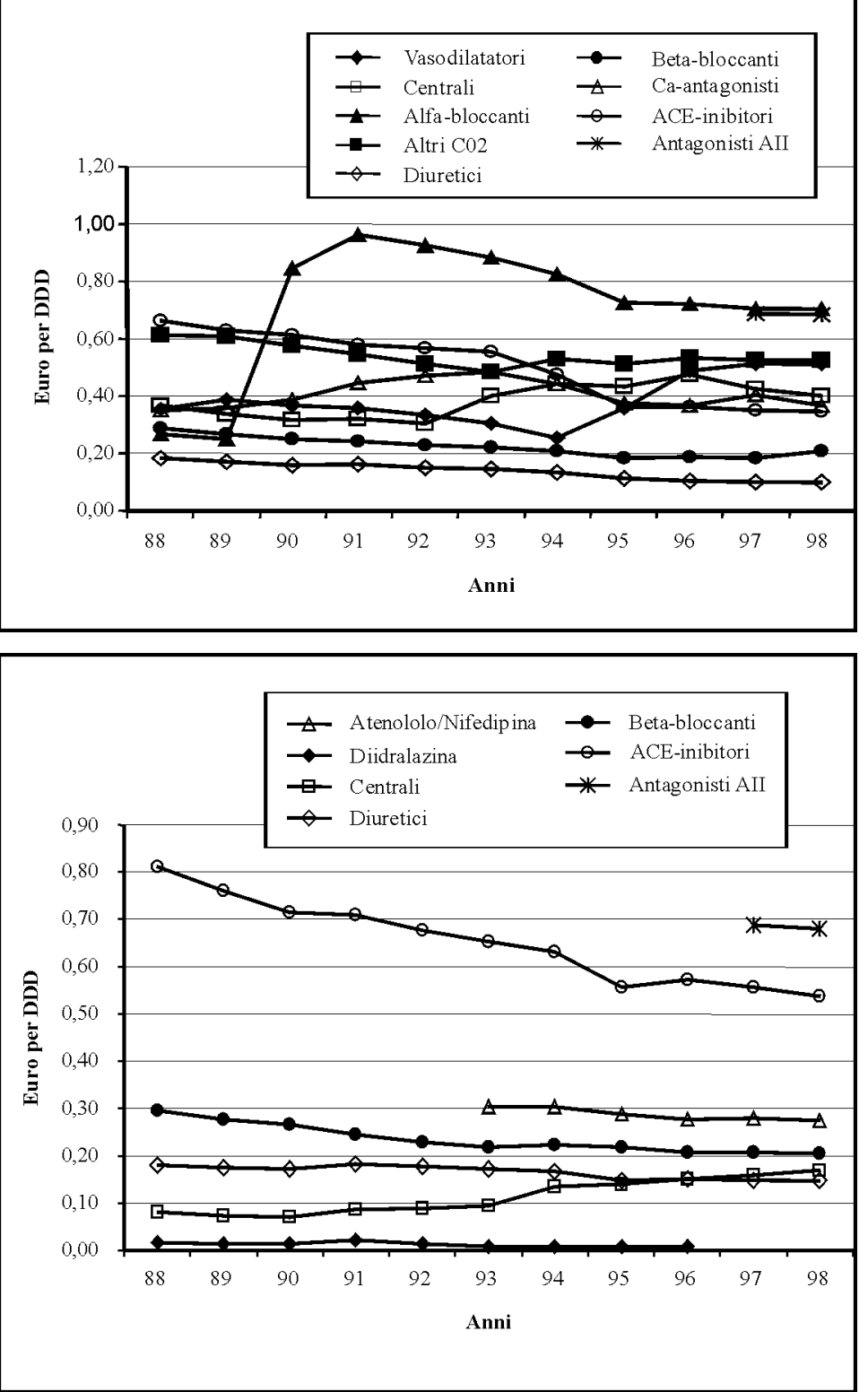

Figura 5a-5b

Andamenti del costo attualizzato per DDD di anti-ipertensivo tra il 1988 e il 1998, separatamente per preparazioni farmaceutiche monocomponente (5a) e in associazione (5b) atto, come dimostrato anche in Italia dalle variazioni delle DDD dei farmaci anti-ipertensivi, che nel decennio preso in esame sono costantemente aumentate di oltre il $10 \%$ all'anno. Tutto fa ritenere che questo andamento sia destinato a continuare nei prossimi anni non solo per il previsto continuo aumento degli ipertesi che vengono posti in trattamento e di quelli trattati efficacemente (cioè con farmaci aggiuntivi o con dosi più elevate), ma anche per i crescenti sforzi volti a ridurre il fenomeno dell'inosservanza. Quest'ultimo aspetto sta diventando di crescente importanza in conseguenza degli effetti negativi che può determinare sulla tutela della salute pubblica, a fronte sia della riduzione della dose necessaria al controllo del fattore di rischio (i.e. l'ipertensione arteriosa), sia dell'interruzione del trattamento in un paziente che continua ad essere esposto al fattore di rischio. Infatti una recente osservazione condotta in Italia ha rivelato le consistenti proporzioni del fenomeno che vede, in un periodo di due anni, circa due terzi dei pazienti interrompere la cura e, tra quelli che continuano ad assumerla periodicamente, una percentuale variabile dal $24 \%$ al $56 \%$ (in base alla classe farmacologica dell' anti-ipertensivo) che la assume ad un dosaggio inferiore a quello utile per la riduzione del rischio cardiovascolare [24].

Le previsioni e le considerazioni sopra esposte, insieme con i dati sulla situazione attuale e sull'andamento della farmacoutilizzazione degli anti-ipertensivi nel recente passato, impongono di riflettere sul peso economico di una strategia di prevenzione farmacologica che attualmente rappresenta il $20 \%$ della spesa farmaceutica pubblica complessiva. Infatti tale peso è destinato a crescere, non solo per l'aumento delle DDD vendute, ma anche a causa del maggior costo delle nuove molecole di anti-ipertensivi immesse in commercio rispetto a quelle già disponibili o che vanno ad essere sostituite.

L'aumento delle DDD nel decennio preso in esame è stato determinato in larghissima parte dagli ACE-inibitori - sia in preparazione farmaceutica monocomponente sia in associazione - e dai Ca-antagonisti. Tra gli altri antiipertensivi di impiego relativamente diffuso solo i diuretici e i beta-bloccanti hanno presentato un modesto aumento, considerando inoltre che soprattutto i diuretici - e in minor misura beta-bloccanti, ACE-inibitori e Ca-antagonisti - sono utilizzati anche in altre condizioni morbose (insufficienza cardiaca, angina e, per i diuretici, tutte le affezioni caratterizzate da ritenzione idrica).

Di particolare rilievo, infine, è l'utilizzazione degli antagonisti AII. Questi farmaci, entrati in commercio nel 1997 e caratterizzati da un 
costo per DDD particolarmente elevato, hanno mostrato nell' anno successivo un aumento di circa il $58 \%$, aumento che è stato di oltre 7 volte per le associazioni con diuretico, confermando la tendenza alla prescrizione dei farmaci di nuova immissione in commercio.

Indipendentemente da considerazioni legate al marketing di un farmaco, un aspetto rilevante in termini di appropriatezza d'uso è rappresentato dal limitato aumento del numero di DDD per diuretici, che in tutto il mondo rappresentano i farmaci di impiego iniziale nell'ipertensione: tale aumento è stato pari al $28 \%$ per le preparazioni farmaceutiche monocomponente a base di diuretico e al $36 \%$ per i preparati in associazione con altri farmaci antiipertensivi; ciò a fronte di un aumento delle DDD del $186 \%$ e del $131 \%$ rispettivamente per ACE-inibitori e per Ca-antagonisti non associati. Questi dati stanno ad indicare che anche in una rilevante percentuale di "nuovi" ipertesi il primo trattamento è stato attuato con altre molecole.

D'altronde tale scenario, che sembra dipendere da strategie promozionali dei farmaci, potrebbe essere conseguente anche ad una maggiore tollerabilità di alcune tra le più recenti molecole che, associandosi per questo ad un minor tasso di interruzione del trattamento, permettono una più rapida ed efficiente gestione del paziente iperteso [25].

In sintesi, le conseguenze del profilo di farmacoutilizzazione fin qui descritto sono illustrate dalla Figura 2, nella quale - accanto al forte aumento delle DDD per anti-ipertensivi si rileva che la corrispondente spesa aumenta con un tasso superiore a circa il $50 \%$ rispetto alle DDD vendute. Questo risultato desta qualche perplessità quando viene valutato sulla base delle evidenze emerse dai trial clinici randomizzati, sia per quanto attiene all'appropriatezza d'uso dei farmaci che all'efficienza dell'investimento economico per la terapia anti-ipertensiva. Infatti gli studi sperimentali evidenziano che i vantaggi del trattamento anti-ipertensivo sono in rapporto con la dimensione della riduzione della pressione arteriosa, più che con il tipo di farmaco utilizzato per ottenerla, residuando differenze tra le diverse categorie di farmaci che riguardano esclusivamente la qualità di vita più che la sopravvivenza. Il recentissimo Studio ALLHAT [26], condotto in 623 Centri in USA e in Canada, su 33.357 ipertesi, seguiti per circa 5 anni, ha dimostrato che il trattamento con clortalidone (come rappresentativo della classe dei diuretici), eventualmente associato con atenololo, clonidina o reserpina, è più efficace di quello con lisinopril (come rappresentativo della classe ACE-inibitori) e di quello con amlodipina (per i Ca-antagonisti), sia in termini di normalizzazione della pressione arteriosa, sia di prevenzione delle complicanze cardiovascolari e di riduzione della letalità.

L'algoritmo per il trattamento dell'ipertensione proposto dal JNC 2003 [5] è in accordo con quanto sopra riportato: negli ipertesi in stadio 1 nei quali non coesiste una indicazione clinica preferenziale alla scelta di un particolare farmaco, è consigliato in prima istanza di iniziare il trattamento con un diuretico tiazidico; negli ipertesi in stadio 2 è consigliata la somministrazione di un diuretico tiazidico associato ad un ACE-inibitore, di un antagonista AII, di un beta-bloccante o di un Ca-antagonista. Solo in presenza di una indicazione preferenziale, la scelta dell'anti-ipertensivo deve essere effettuata nel rispetto di tale indicazione: per esempio, in un paziente coronaropatico dovrà essere preferito un beta-bloccante o un Ca-antagonista. Una oculata applicazione di queste regole avrebbe certamente delle conseguenze positive dal punto di vista della spesa: come desumibile dalla Figura 1, il costo annuo per paziente del trattamento antiipertensivo varia profondamente tra le diverse classi di farmaci: dai 10-50 euro per i diuretici agli 80-250 euro per i beta-bloccanti, ai 150-300 euro per gli ACE-inibitori, ai 220-380 euro per i Ca-antagonisti fino ai 300-450 euro per gli antagonisti AII.

In conclusione, tale analisi storica ha evidenziato un profondo cambiamento della farmacoutilizzazione degli anti-ipertensivi in Italia durante l'ultimo decennio del secolo scorso, derivante da una maggiore consapevolezza sociale del fattore di rischio "ipertensione arteriosa" a cui ha corrisposto un crescente impatto economico della terapia antiipertensiva sia come valore assoluto, sia rispetto alla spesa farmaceutica complessiva.

\section{RINGRAZIAMENTI}

Il presente studio è stato realizzato sulla base delle linee di ricerca farmacoeconomiche proposte dalla Società Italiana per studi di Economia ed Etica sul Farmaco e sugli Interventi Terapeutici. Gli autori e la SIFEIT sono grati ad IMS-Health per aver messo a disposizione la base dati necessaria allo sviluppo del presente studio. In particolare si ringrazia la dottoressa Franca Bottacin di IMS-Health e la dottoressa Rosalia Galasso per la gentile collaborazione. In considerazione sia delle finalità etiche e sociali del presente articolo, sia della assenza di intenti diretti a promuovere o a contrastare l'uso di un farmaco in particolare, gli autori dichiarano la assoluta mancanza di conflitto di interessi sia nella progettazione dello studio che nella sua stesura. 


\section{BIBLIOGRAFIA}

1. ISTAT-Istituto Nazionale di Statistica. Il quadro evolutivo della mortalità per causa in Italia: anni 1991, 1994, 1997. http:/ /www.istat.it/ (aggiornato al 28 Maggio 2003)

2. Hilleman DE, Mohjuddin SM, Lucas BD et al. Cost-minimization analysis of initial antihypertensive therapy in patients with mild-to-moderate essential diastolic hypertension. Clin Ther 1994:16,88.

3. Wolf-Maier K, Cooper RS, Banegas JR et al. Hypertension prevalence and blood pressure levels in 6 European Countries, Canada and the United States JAMA 2003; 289, 2363

4. The Sixth Report of the Joint National Committee on Prevention, Detection, Evaluation and Treatment of High Blood Pressure. Arch Intern Med 1997:157,3.

5. The Seventh Report of the Joint National Committee on Prevention, Detection, Evaluation and Treatment of High Blood Pressure. JAMA 2003:289,2560

6. Franklin SS, Gustin W, Wong ND et al. Hemodynamic patterns of age-related changes in blood pressure. Circulation 1997:96,308

7. Guidelines Subcommittee. 1999 World Health Organisation - International Society of Hypertension: Guidelines for the management of hypertension. J Hypertens 199917,151

8. SHEP Cooperative Research Group: Prevention of stroke by antihypertensive drug treatment in older persons with isolated systolic hypertension. Final results of the Systolic Hypertension in the Elderly Program (SHEP). JAMA 1991:265,3255

9. Staessen JA, Fagard R, Thijs L et al. Randomized double-blind comparison of placebo and active treatment for older patients with isolated systolic hypertension. The Systolic Hypertension in Europe (Syst-Eur) Trial Invesrtigators. Lancet 1997:350,757

10. Liu L, Wang JG, Gong L et al. Comparison of active treatment and placebo in older Chinese patients with isolated systolic hypertension. Systolic Hypertension in China (Syst-China) Collaborative Group. J Hypertens 1998:16 (Part 1), 1823

11. Amery A, Birkenhager W, Brixko P et al. Mortality and morbidity results from the European Working Party on High Blood Pressure in the Elderly trial. Lancet 1985:1,1349

12. Dahlof B, Lingholm LH, Hansson L et al. Morbidity and mortality in the Swedish Trial in Old Patients with Hypertension (STOP-Hypertension). Lancet 1991:338,1281

13. Medical Research Council trial of treatment of hypertension in older adults: principal results. BMJ 1992:304,405

14. Gong L, Zhang W, Zhu Y et al. Shanghai Trial of Nifedipine in the Elderly (STONE). J Hypertens 1996:14,1237

15. Gueyffier F, Bulpitt C, Boissel JP et al. Antihypertensive drugs in very old people: a subgroup meta-analysis of randomised controlled trials. Lancet 1999:353,793

16. Leonetti G, Cuspidi C, Facchini M La terapia antiipertensiva negli anziani: I risultati dei grandi trial. Ital Hearth J Suppl 2001:2,1161

17. McMurray J. The health economics of the treatment of hyperlipidemia and hypertension. Am J Hypertens 1999:12,99S

18. Degli Esposti L, Capone A, Degli Esposti E et al. Analisi della persistenza e delle risorse allocate nel trattamento farmacologico dell'ipertensione arteriosa. Farmaeconomia e Percorsi Terapeutici 2002; 3(3), 161

19. Ministero della Salute. L'uso dei farmaci in Italia. Rapporto Nazionale, Anno 2002. Roma: Osservatorio Nazionale sull'impiego dei Medicinali (OSMED), 2003

20. IMS-Health Italia. Il mercato farmaceutico. Milano: IMS, 1988-1998.

21. Farmindustria. La spesa farmaceutica in Italia: trend storico. In: Fatti e Cifre 2003 (pp.37). Roma: Farmindustria, 2003

22. Eandi M. Unità di consumo dei farmaci e valutazioni farmacoeconomiche: uso e misuso di DDD e PDD. Farmaeconomia e Percorsi Terapeutici 2002; 3(4), 209

23. Martini N. Nuovo prontuario farmaceutico e diritti dei cittadini. Recenti Progressi in Medicina 1994; 85(5), 267

24. Degli Esposti E, Sturani A, Degli Esposti Let al. Pharmacoutilization of antihypertensive drugs: a model of analysis. Internat J Clin Pharmacol Ther 2001:39,251

25. Degli Esposti E, Sturani A, Di Martino M, Falasca P, Novi MV, Baio G, et al. Long-term persistence with antihypertensive drugs in new patients. J Hum Hypertens 2002; 16, 439-444

26. ALLHAT Collaborative Research Group. Major outcomes in high-risk hypertensive patients randomized to angiotensin-converting-enzyme inhibitor or calcium channel blocker vs diuretic: The Antihypertensive and Lipid Lowering Treatment to prevent Heart Attack (ALLHAT). JAMA 2002; 288, 2981 